\title{
Relational Abstractions for Continuous and Hybrid Systems ${ }^{\star}$
}

\author{
Sriram Sankaranarayanan ${ }^{1}$ and Ashish Tiwari $^{2}$ \\ 1 University of Colorado, Boulder, CO \\ srirams@colorado.edu \\ 2 SRI International, Menlo Park, CA \\ ashish.tiwari@sri.com
}

\begin{abstract}
In this paper, we define relational abstractions of hybrid systems. A relational abstraction is obtained by replacing the continuous dynamics in each mode by a binary transition relation that relates a state of the system to any state that can potentially be reached at some future time instant using the continuous dynamics. We construct relational abstractions by reusing template-based invariant generation techniques for continuous systems described by Ordinary Differential Equations (ODE). As a result, we abstract a given hybrid system as a purely discrete, infinite-state system. We apply k-induction to this abstraction to prove safety properties, and use bounded model-checking to find potential falsifications. We present the basic underpinnings of our approach and demonstrate its use on many benchmark systems to derive simple and usable abstractions.
\end{abstract}

\section{Introduction}

In this paper, we present relational abstractions of hybrid systems. A relational abstraction transforms a given hybrid system into a purely discrete transition system by summarizing the effect of the continuous evolution of states over time using relations. The abstract discrete system is an infinite-state system that can be analyzed using standard techniques for verifying systems such as $k$-induction and bounded model checking.

Relational abstractions preserve the discrete behavior of the hybrid system and abstract only its continuous behavior. They work by replacing the continuous dynamics in each mode by means of a relation $R\left(\boldsymbol{x}_{0}, \boldsymbol{x}\right)$. The relation $R$ relates a continuous state $\boldsymbol{x}_{0}$ with a state $\boldsymbol{x}$ that can be potentially reached at some future time instant, through some time trajectory of the system starting from $\boldsymbol{x}_{0}$. Such a relation $R$ can be interpreted in two ways: (a) as a positive invariant set for an associated dynamical system over $\boldsymbol{x}_{0}, \boldsymbol{x}$, and (b) as a discrete transition relation that abstracts the evolution of the continuous states over time.

\footnotetext{
* Sankaranarayanan's work has been supported by NSF Career grant CNS-0953941. Tiwari's work supported in part by DARPA under Contract No. FA8650-10-C-7078, NSF grants CSR-0917398 and SHF:CSR-1017483.
}

G. Gopalakrishnan and S. Qadeer (Eds.): CAV 2011, LNCS 6806, pp. 686-702, 2011.

(C) Springer-Verlag Berlin Heidelberg 2011 
The two views above provide two key advantages of the relational abstraction approach. As a consequence of the first view, we can use techniques for generating invariants for continuous systems to generate a relational abstraction. We propose simple extensions of template-based invariant generation techniques, which can abstract systems with linear as well as nonlinear dynamics, to construct relational abstractions. Template-based techniques allow us to specify the form of the relational abstraction [9]19. Therefore, our technique can be used to obtain linear arithmetic relations for systems with nonlinear dynamics.

As a consequence of the second view, we obtain discrete infinite-state abstractions of hybrid systems. This enables us to use techniques such as $k$-induction using decision procedures [37, abstract interpretation [1121], or virtually any technique for discrete systems, to analyze hybrid systems.

It is well-known that the problem of verifying hybrid systems is quite hard, both in theory and in practice. Recently, many advances have yielded remarkably efficient tools for integrating affine ODEs over sets and that work over large state spaces 24/539]16/35]17. However, we have observed that a significant gap in performance remains when these techniques are used to perform symbolic model checking, along the lines of tools such as HyTech and PHAVer [23[15. In our experience, this gap stems from the need to handle the dynamics repeatedly for the same mode, often with small variations between subproblems. In this paper, we hypothesize that the situation with continuous dynamics is analogous to that of function calls encountered during program analysis. During inter-procedural program analysis, it is often observed that the analysis of each function call, given the state at the entry to the call, is quite efficient. However, the overall inter-procedural analysis is often not scalable due to repeated analysis of the same function with different actual parameters. Therefore, as in the case of function calls in program analysis, we propose summarization techniques that abstract the effect of the dynamics in each mode by a discrete transition [27]. As a result, our technique can efficiently handle continuous dynamics. However, on the flip side, our approach may lose precision if the relational abstraction is too conservative. Furthermore, the computation of relational invariants implicitly doubles the number of state variables.

Our approach is able to prove safety properties of hybrid systems using techniques such as $k$-induction, as well as to discover potential violations through bounded model checking. To evaluate the idea of using relational abstractions of hybrid systems, we generate relational abstractions of some standard benchmarks and model check these abstractions. We generate relational abstractions using a combination of quantifier elimination tools (REDLOG, QEPCAD) to search for templatized invariants 42838 , and polyhedral analysis of ODEs using fixed point iteration over cones [36]. We analyze the resulting relational abstractions using the SAL framework from SRI [34 40]. Our preliminary experiments are quite promising: our approach has the ability to prove properties of hybrid systems that are known to be complex, while at the same performing much more efficiently than symbolic model checkers. The data from our exper- 
iments along with an extended version of this paper with proofs will be made available online1. We now discuss other related ideas in the literature.

Transition Invariants and Variance Analysis: The idea of defining "progress" invariant predicates over pairs of states, $\mathrm{x}, \overline{\mathrm{x}}$, is well-known in the field of program analysis. A lot of work has been done on verifying liveness properties using ranking functions, transition invariants, and progress invariants 4301018 . However, there are some important distinctions between these various forms of relational invariants. Transition invariants [30] capture the relationship between the current state and any previous state (at a particular program location). Transition invariants were used to prove termination. Progress invariants capture the relationship between the current state and the immediately previous state (at a particular program location) [18]. Progress invariants were used to compute complexity bounds of programs. The relational abstractions presented here have a subtle difference: they capture the relationship between the current state and all previous states after the current mode was entered. When combined with the entry and exit conditions of a mode, relational invariants are exactly summaries of that mode. We use relational invariants to create abstractions of hybrid systems that can be used, for instance, to verify safety properties.

Podelski and Wagner provide a verification procedure for (region) stability properties of hybrid systems [31, where they derive binary reachability relations over trajectories of a hybrid system, similar to what is being proposed here. However, there are two key differences in our methodology: (a) Our approach deals with the dynamics at each mode up front, deriving relational abstractions. On the other hand, the technique of Podelski et al. transforms the entire hybrid system, relying on safety verification built into a tool such as Phaver to derive the relations [15]. Our goal in this paper is to make the process more efficient using constraint-based approaches and improve hybrid system safety verification in the first place. (b) Second, our ultimate goal is to verify safety properties efficiently as opposed to verifying stability.

Abstractions of Hybrid Systems: Many different types of discrete abstractions have been studied for hybrid systems including predicate abstraction [2]41] and abstractions based on invariants 28. The use of counterexample-guided abstraction refinement for iterative refinement has also been investigated in the past (Cf. Alur et al. 2] and Clarke et al. 7], for example). In this paper, the proposed abstraction yields a discrete but infinite state system.

Hybridization is a technique for converting nonlinear systems into affine systems by subdividing the invariant region into numerous subregions and approximating the dynamics as a hybrid system by means of a linear differential inclusion in each region 23312 . However, such a subdivision is expensive as the number of dimensions increases and often infeasible if the invariant region is unbounded.

Reachability Analysis: Reasoning about the reachable set of states for flows of nonlinear systems is an important primitive that is used repeatedly in the

${ }^{1}$ Cf. http://www.csl.sri.com/ tiwari/relational-abstraction/ 
analysis of nonlinear hybrid systems. This has been addressed using a wide variety of techniques in the past, including algebraic techniques, interval analysis, constraint propagation, and Bernstein polynomials [32 2629|3313].

\section{Preliminaries}

We present the basic definitions and properties of continuous systems defined by Ordinary Differential Equations (ODE). Let $\mathbb{R}$ denote the set of real numbers. We use $\boldsymbol{a}, \ldots, \boldsymbol{z}$ with subscripts to denote (column) vectors and $A, \ldots, Z$ to denote matrices. For an $m \times n$ matrix $A$, the row vector $A_{i}$, for $1 \leq i \leq m$, denotes the $i^{\text {th }}$ row. We define continuous systems using vector fields.

Definition 1 (Vector Field). A vector field $\mathfrak{F}$ over a set $X \subseteq \mathbb{R}^{n}$ is a function $\mathfrak{F}: X \mapsto \mathbb{R}^{n}$ mapping each $\boldsymbol{x} \in X$ with a field direction $\mathfrak{F}(\boldsymbol{x})$.

Vector fields commonly arise from the definition of time-invariant systems. A time-invariant system defined by the ODE $\frac{d x_{1}}{d t}=f_{1}(\boldsymbol{x}), \ldots, \frac{d x_{n}}{d t}=f_{n}(\boldsymbol{x})$ can be identified with the vector field $\mathfrak{F}(\boldsymbol{x})=\left(f_{1}(\boldsymbol{x}), \ldots, f_{n}(\boldsymbol{x})\right)$. Therefore, a continuous system $\mathcal{S}:\langle\mathfrak{F}, X\rangle$ is defined by a tuple consisting of the vector field $\mathfrak{F}$ and a domain (also referred to as a mode invariant) $X \subseteq \mathbb{R}^{n}$. We now define the time trajectories of a continuous system:

Definition 2 (Time Trajectories). A time trajectory of a continuous system $\mathcal{S}:\langle\mathfrak{F}, X\rangle$ is a function $\tau:[0, T) \mapsto \mathbb{R}^{n}$ for some $T>0$, such that: $\tau(t) \in X$, for all $t \in[0, T)$ and $\frac{d \tau}{d t}=\mathfrak{F}(\tau(t)), \forall t \in[0, T)$.

Note 1. To facilitate presentation, we have (deliberately) restricted our attention to time-invariant and autonomous systems. The full generalization to time variant, non-autonomous systems will be presented in an extended version.

If the continuous system $\mathcal{S}$ is defined by a Lipschitz continuous vector field $\mathfrak{F}$, then for any $\boldsymbol{x}_{0} \in X$, we can guarantee the existence of a unique time trajectory $\tau$ such that $\tau(0)=\boldsymbol{x}_{0}$ [25. Henceforth, we will assume that the systems considered are defined by Lipschitz continuous vector fields. An affine system $\mathcal{S}$ is a continuous system whose dynamics are defined by an affine vector field $\frac{d \boldsymbol{x}}{d t}=A \boldsymbol{x}+\boldsymbol{b}$. If $f(\boldsymbol{x})$ is continuous and differentiable over $\boldsymbol{x}$ then we write $\partial_{\boldsymbol{x}} f$ to denote the vector of its partial derivatives w.r.t each $\boldsymbol{x}_{i}$. The Lie derivative of a function $g$ with respect to a field $\mathfrak{F}$ is given by $\mathcal{L}_{F}(g):=\left(\partial_{\boldsymbol{x}} g\right) \cdot \mathfrak{F}(\boldsymbol{x})$, where '.' computes the dot product of two vectors.

Positive Invariant Set: A set $M \subseteq X$ is an invariant set for the system $\mathcal{S}$ iff for any $\boldsymbol{x} \in M$, every time trajectory $\tau:[0, T) \mapsto X$ such that $\tau(0)=\boldsymbol{x}$ is entirely contained in $M$; that is, $(\forall t \in[0, T)) \tau(t) \in M$.

Let $M$ be a closed set defined by the assertion $\bigwedge_{j=1}^{m} g_{j}(\boldsymbol{x}) \leq 0$ for some finite $m$. For technical reasons, we assume that each $g_{j}(\boldsymbol{x})$ is continuous and differentiable, and $M$ is a "practical set" satisfying the constraint qualification (Cf. Blanchini \& Miani [6], page 104):

$$
(\forall \boldsymbol{x} \in X),(\exists \boldsymbol{z}) g_{j}(\boldsymbol{x})+\partial_{\boldsymbol{x}} g_{j} \cdot \boldsymbol{z}<0 .
$$




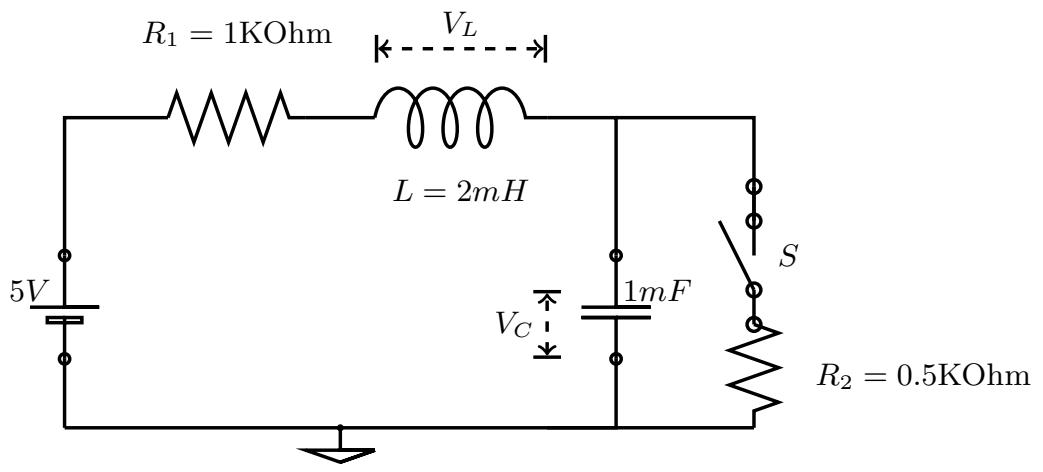

Fig. 1. Circuit diagram for an LCR circuit with a voltage-controlled switch $S$

Informally, the constraint qualifications ensure that $\bigwedge_{j} g_{j}(\boldsymbol{x})<0$ represents the (relative) interior of the set $M$ and $\bigvee_{j} g_{j}(\boldsymbol{x})=0$ represents the boundary. It can be shown that all affine functions $g_{j}$ and positive-semidefinite quadratic forms (defining $n$-dimensional ellipsoids) satisfy these conditions.

Theorem 1. The set $M: \bigwedge_{j=1}^{m} g_{j}(\boldsymbol{x}) \leq 0$ is a positive invariant for the vector field $\mathfrak{F}$ if for each $j \in[1, m]$ the following assertion holds true: $\forall \boldsymbol{x} \in X: g_{j}(\boldsymbol{x})=$ $0 \wedge \bigwedge_{i \neq j} g_{i}(\boldsymbol{x}) \leq 0 \Rightarrow \mathcal{L}_{F}\left(g_{j}\right)<0$.

The theorem states that under appropriate conditions, a closed set $M$ is a positive invariant set if the vector field $\mathfrak{F}$ lies in the tangent cone at each point on the boundary of the set. It is a direct consequence of Nagumo's theorem, a more general result that holds for non-Lipschitz continuous dynamics and non- "practical" sets as well. The theorem above provides a basis for various techniques for generating invariants for continuous systems using quantifier elimination and constraint solving 32361929 .

Hybrid Systems: Hybrid systems combine the continuous evolution of state with discrete, instantaneous jumps that can alter the state as well as the dynamics of a system [22].

Definition 3 (Hybrid System). A hybrid system $\mathcal{H}$ is defined by a set of discrete modes $\left\langle m_{1}, \ldots, m_{k}\right\rangle$, wherein, each mode $m_{i}$ is defined by a continuous system $\mathcal{S}_{i}:\left\langle\mathfrak{F}_{i}, X_{i}\right\rangle$. The system can change modes through a set of discrete transitions $\tau_{1}, \ldots, \tau_{m}$. Each transition is defined by a prior mode $m_{0}$, a postmode $m_{1}$ and a transition relation $\rho\left[\boldsymbol{x}, \boldsymbol{x}^{\prime}\right] \subseteq X_{m_{0}} \times X_{m_{1}}$, that relates the state $\boldsymbol{x} \in X_{m_{0}}$ before the transition to the state $\boldsymbol{x}^{\prime} \in X_{m_{1}}$ obtained as a result of taking the transition. The initial conditions are given by the initial mode $m_{\text {init }}$ with the initial state set $\Theta \subseteq X_{\text {init }}$.

A hybrid system is a switched system if each discrete transition of the system does not modify the continuous state variables. In other words, each discrete transition relation $\rho\left[\boldsymbol{x}, \boldsymbol{x}^{\prime}\right]$ can be written as $\rho: \gamma(\boldsymbol{x}) \wedge \boldsymbol{x}^{\prime}=\boldsymbol{x}$, for guard $\gamma(\boldsymbol{x})$. 
Example 1 (Switched system). Figure1 shows the circuit diagram for a voltagecontrolled switch that closes whenever the voltage across the capacitor $\left(V_{C}\right)$ exceeds $4 V$, and opens whenever $V_{C}$ goes below $1 V$.

With the switch $S$ open, the dynamics of the voltage across capacitor $V_{C}$ and the voltage across the inductor $V_{L}$ are given by $\frac{d V_{C}}{d t}=5-V_{C}-V_{L}, \quad \frac{d V_{L}}{d t}=$ $-5+V_{C}-V_{L}$. Likewise, with the switch $S$ closed, the dynamics of the voltage across the inductor are given by $\frac{d V_{C}}{d t}=5-3 V_{C}-V_{L}, \quad \frac{d V_{L}}{d t}=-5+3 V_{C}-V_{L}$. In each mode, we assume the mode invariant $\left(V_{C}, V_{L}\right) \in[-10,10] \times[-10,10]$.

\section{Relational Abstractions}

We define relational abstractions for continuous systems, and present proof rules for checking that a given relation is an abstraction of the time trajectories of a continuous system defined by ODEs.

Let $\mathcal{S}:\langle\mathfrak{F}, X\rangle$ be a continuous system defined by the vector field $\mathfrak{F}$, and domain (invariant) $X$. It is assumed that $\mathcal{S}$ arises from a mode of a larger hybrid system. Let $R(\boldsymbol{x}, \boldsymbol{y})$ be a relation over $X \times X$.

Definition 4 (Relational Abstraction Without Time). The relation $R \subseteq$ $\mathbb{R}^{2 n}$ is a (timeless) relational abstraction of a continuous system $\mathcal{S}$ if for all time trajectories $\tau:[0, T) \mapsto X$ of the system $\mathcal{S}$, it is the case that $(\forall t \in$ $[0, T))(\tau(0), \tau(t)) \in R$.

Thus, for a time invariant system, a relational abstraction $R$ captures all pairs of states $(\boldsymbol{x}, \boldsymbol{y})$ such that it is possible to reach $\boldsymbol{y}$ from $\boldsymbol{x}$ in a finite amount of time by evolving according to the dynamics of the system.

A relational abstraction $R \subseteq X \times X$ is said to be complete for a system $\mathcal{S}$ if whenever $R(\boldsymbol{x}, \boldsymbol{y})$ holds, there exists a time trajectory $\tau:[0, T) \mapsto X$ such that $\tau(0)=\boldsymbol{x}$ and $\tau(t)=\boldsymbol{y}$, for some time $0 \leq t<T$. Likewise, a relational abstraction $R$ is linear if it can be expressed as an assertion in the theory of linear arithmetic over reals.

Note 2. A continuous system whose dynamics are defined by constants (such as a mode of a multirate hybrid automaton) has a complete, linear relational abstraction. For instance, the evolution of the ODE $\frac{d x}{d t}=2, \frac{d y}{d t}=-3$ can be abstracted by the relation $R\left(x, y, x^{\prime}, y^{\prime}\right):=x^{\prime}-x \geq 0 \wedge \frac{1}{2}\left(x^{\prime}-x\right)=\frac{-1}{3}\left(y^{\prime}-y\right)$. In fact, we can show that hybrid systems with constant dynamics in each mode are bisimilar to a purely discrete transition system through relationalization. On the other hand, linear vector fields can fail to have complete abstractions.

We now define an "extended system" $\mathcal{S}^{\prime}$ from a given system $\mathcal{S}$ such that invariants of $\mathcal{S}^{\prime}$ will yield relational abstractions for $\mathcal{S}$.

Definition 5 (Extended System). Let $\mathcal{S}$ be a continuous system over $\boldsymbol{x} \in \mathbb{R}^{n}$ defined by vector field $\mathfrak{F}$ and invariant region $X$. The extended system $\mathcal{S}^{\prime}$ has state variables $(\boldsymbol{x}, \boldsymbol{y}) \in \mathbb{R}^{2 n}$ with the dynamics.

$$
\frac{d \boldsymbol{y}}{d t}=\mathfrak{F}(\boldsymbol{y}), \frac{d \boldsymbol{x}}{d t}=\mathbf{0},
$$


invariant region given by $X \times X$ and with the initial conditions $\boldsymbol{x}(0)=\boldsymbol{y}(0) \in X$.

We now refine the notion of positive invariants from Section 2 to account for the presence of initial conditions in the system.

Definition 6 (Initialized Positive Invariant). A set $M$ is an initialized positive invariant for the system $\mathcal{S}$ with initial conditions $X_{0} \subseteq M$ iff for all time trajectories $\tau:[0, T) \mapsto \mathbb{R}^{n}$ of $\mathcal{S}$ starting from $\tau(0) \in X_{0}$ we have $\tau(t) \in M$ for all $t \in[0, T)$.

An initialized positive invariant is an over-approximation of all states reachable through a time trajectory starting from some pre-specified set of initial states. This is, in fact, the true analog of an invariant for a program.

Note that every positive invariant set $M$ (following the definition in Section 2) that contains the initial set $X_{0}$ is an initialized positive invariant. On the other hand, an initialized positive invariant may not be a general positive invariant. This is because, it may be possible for trajectories that start from some state in the set $M-X_{0}$ to exit the invariant set $M$.

Lemma 1. A relation $R$ is a relational abstraction of $\mathcal{S}$ if and only if $R$ is an initialized positive invariant for $\mathcal{S}^{\prime}$.

Proofs are provided in an extended version of the paper.

Therefore, if we can compute initialized positive invariants of the extended system $\mathcal{S}^{\prime}$ with initial states $\boldsymbol{x}(0)=\boldsymbol{y}(0)$, we may use them to obtain relational abstractions. In this work, we use various techniques that can compute positive invariants $M$ (using the definition in Section 2) of systems $\mathcal{S}$ that contain some initial set of states $X_{0}$.

Theorem 2. Let $M$ be a positive invariant of the extended system $\mathcal{S}^{\prime}$ containing the initial states $X_{0}=\{(\boldsymbol{x}, \boldsymbol{x}) \mid \boldsymbol{x} \in X\}$. Then $M$ is a relational abstraction of the system $\mathcal{S}$.

Proof. We note that a positive invariant $M$ containing the initial states $X_{0}$ is also an initialized positive invariant. I.e, for any trajectory $\sigma$ starting from $X_{0}$, we know that $\sigma(t) \in M$ since $\sigma(0) \in M$. The rest follows from Lemma 1 .

The converse of the theorem above does not hold, in general. As discussed above, a positive invariant $M$ containing the initial set of states $X_{0}$ is not necessarily an initialized positive invariant.

Note 3. The extended system can be expressed, equivalently, using the (time reversed) system instead of the system (2),

$$
\frac{d \boldsymbol{y}}{d t}=\mathbf{0}, \frac{d \boldsymbol{x}}{d t}=-\mathfrak{F}(\boldsymbol{x})
$$

with the initial conditions $\boldsymbol{x}(0)=\boldsymbol{y}(0)$.

In other words, a relational abstraction $R(\boldsymbol{x}, \boldsymbol{y})$ is a positive invariant of one of two dynamical systems: System 2 where $\boldsymbol{x}$ is frozen in time and $\boldsymbol{y}$ evolves 
according to the vector field $\mathfrak{F}$, and System 3 where $\boldsymbol{y}$ is frozen in time and $\boldsymbol{x}$ evolves according to the time reversed field $-\mathfrak{F}$.

Proof Rule for Relational Abstractions: The proof rule for relational abstractions can be derived from the proof rule for invariant sets. Furthermore, techniques for synthesizing invariants can be directly used to synthesize relational abstractions. We now present a proof rule for checking if a relation $R$ is a sound abstraction. We assume that the relation $R$ is specified as an assertion of the form

$$
R(\boldsymbol{x}, \boldsymbol{y}): g_{1}(\boldsymbol{x}, \boldsymbol{y}) \leq 0 \wedge \ldots \wedge g_{m}(\boldsymbol{x}, \boldsymbol{y}) \leq 0,
$$

wherein $g_{1}, \ldots, g_{m}$ are continuous and differentiable functions over $\mathbb{R}^{2 n}$. Furthermore, for technical reasons, we assume that the set $R \cap(X \times X)$ in $\mathbb{R}^{2 n}$ defined by the relation $R$ restricted to $X$ is a closed set and $g_{j}$ satisfy the constraint qualifications in (1).

Definition 7. The following rules allow us to conclude that the relation $R$, as specified above, is a relational abstraction of a continuous system $\mathcal{S}$ :

Initialization: $\forall \boldsymbol{x} \in X, R(\boldsymbol{x}, \boldsymbol{x})$, and

Flow Preservation: We may use the rule for forward time:

$$
\forall j \in[1, m], \forall \boldsymbol{x}, \boldsymbol{y} \in X, \bigwedge_{\dot{\dot{H}^{\prime}}} g_{i}(\boldsymbol{x}, \boldsymbol{y}) \leq 0 \wedge g_{j}(\boldsymbol{x}, \boldsymbol{y})=0 \Rightarrow\left(\partial_{\boldsymbol{y}} g_{j}\right) \cdot \mathfrak{F}(\boldsymbol{y})<0,
$$

or the rule for time reversed dynamics:

$$
\forall j \in[1, m], \forall \boldsymbol{x}, \boldsymbol{y} \in X, \bigwedge_{\dot{\forall}\langle j} g_{i}(\boldsymbol{x}, \boldsymbol{y}) \leq 0 \wedge g_{j}(\boldsymbol{x}, \boldsymbol{y})=0 \Rightarrow\left(\partial_{\boldsymbol{x}} g_{j}\right) \cdot(-\mathfrak{F}(\boldsymbol{x}))<0 .
$$

Example 2. We now consider relationalizations for the inductor-capacitorresistor (LCR) circuit in Example 1. Consider the mode when the switch is open with dynamics given by $\frac{d V_{C}}{d t}=5-V_{C}-V_{L}, \quad \frac{d V_{L}}{d t}=-5+V_{C}-V_{L}$.

We wish to show that the relation $R\left(V_{C 0}, V_{L 0}, V_{C}, V_{L}\right)$, represented by the assertion below, is a relational abstraction: $\left(V_{C 0}, V_{C}, V_{L 0}, V_{L}\right) \in[-10,10]^{4} \wedge$ $V_{C}+5 V_{L} \leq V_{C 0}+50 \wedge 4 V_{L} \leq V_{L 0}+30 \wedge 2 V_{L}-3 V_{C} \leq 2 V_{L 0}+30$.

Let us consider the inequality $V_{C}+5 V_{L}-V_{C 0}-50 \leq 0$. For the initial condition, we set $V_{C}=V_{C 0}$ and $V_{L}=V_{L 0}$ and verify that $5 V_{L 0} \leq 50$ holds over the invariant region $\left(V_{C 0}, V_{L 0}\right) \in[-10,10]^{2}$. Likewise, the Lie derivative of the left-hand side expression is given by $4 V_{C}-6 V_{L}-20$. We verify the following entailment using an SMT solver

$$
R\left(V_{C 0}, V_{L 0}, V_{C}, V_{L}\right) \wedge V_{C}+5 V_{L}-V_{C 0}-50=0 \vDash 4 V_{C}-6 V_{L}-20<0 .
$$

The remaining constraints are similarly verified.

Disjunctive Relational Abstraction: Often, the relational abstraction can be represented as the disjunction $R(\boldsymbol{x}, \boldsymbol{y}): \bigvee_{j=1}^{m} R_{j}(\boldsymbol{x}, \boldsymbol{y})$ of finitely many relations $R_{1}, \ldots, R_{m}$, such that (a) each relation $R_{j}$ is represented by an assertion over $\boldsymbol{x}, \boldsymbol{y}$ satisfying the flow preservation proof rule in Definition 7 , and (b) the disjunctive relation $R(\boldsymbol{x}, \boldsymbol{y})$ satisfies the initialization rule. 
Example 3. Consider, once again, the LCR circuit in Example 1. The relation below is a disjunctive relational abstraction for the switch open mode:

$$
\left|V_{L}\right| \leq \max \left(\left|V_{L 0}\right|,\left|V_{C 0}-5\right|\right) \wedge\left|V_{C}-5\right| \leq \max \left(\left|V_{L 0}\right|,\left|V_{C 0}-5\right|\right) .
$$

Verifying this fact can be performed by expanding the definitions of max and $|\cdot|$. The resulting assertion is cast in the disjunctive normal form, and the flow preservation proof rule in Definition 7 can be checked for each disjunct. The initialization rule can be checked for the whole disjunction.

\subsection{Relational Abstractions of Hybrid Systems}

A relational abstraction of a hybrid system is constructed by replacing each constituent continuous system by its relational abstraction and keeping the discrete transitions unchanged. Specifically, if $\mathcal{H}$ is a hybrid system (Definition 3) with $k$ modes and $n$ real-valued variables, then the relational abstraction of $\mathcal{H}$ is a state transition system over the state space $\{1, \ldots, k\} \times \mathbb{R}^{n}$ whose transition relation is the union of the discrete transitions of $\mathcal{H}$ and the relational abstractions of the $k$ modes of $\mathcal{H}$.

Several classes of hybrid automata, such as timed automata and linear hybrid automata, have complete relational abstractions. Since the discrete transitions are not abstracted, we only need to ensure that the relational abstraction of the continuous dynamics are complete.

Timed Automata: The continuous dynamics of a timed automata with $n$ clocks, $x_{1}, \ldots, x_{n}$, can be abstracted by the relation $\wedge_{i=2}^{n}\left(x_{1}-x_{10}=x_{i}-x_{i 0}\right) \wedge$ $x_{1} \geq x_{10}$. It is easy to check that this is a complete abstraction.

Multirate Automata: The continuous dynamics defined by ODEs $\frac{d x_{1}}{d t}=$ $c_{1}, \ldots, \frac{d x_{n}}{d t}=c_{n}$, where $c_{1}, \ldots, c_{n}$ are nonzero constants, can be abstracted by the relation $\wedge_{i=2}^{n}\left(\frac{x_{1}-x_{10}}{c_{1}}=\frac{x_{i}-x_{i 0}}{c_{i}}\right) \wedge \frac{x_{1}-x_{10}}{c_{1}} \geq 0$. Again, it is easy to check that this is a complete abstraction. Note that the result for timed automata is obtained as a special case where all $c_{i}$ are 1 .

Rectangular Automata: In rectangular automata, the dynamics in each mode are specified as $a_{1} \leq \frac{d x_{1}}{d t} \leq b_{1}, \ldots, a_{n} \leq \frac{d x_{n}}{d t} \leq b_{n}$. Assuming $0<a_{i} \leq b_{i}$ for all $i$, these dynamics can be abstracted by the relation

$$
0 \leq \max \left(\frac{x_{1}-x_{10}}{b_{1}}, \ldots, \frac{x_{n}-x_{n 0}}{b_{n}}\right) \leq \min \left(\frac{x_{1}-x_{10}}{a_{1}}, \ldots, \frac{x_{n}-x_{n 0}}{a_{n}}\right)
$$

Again, it is easy to check that this is a complete abstraction.

Linear Hybrid Automata: In linear hybrid automata, the dynamics in each mode are specified as a linear constraint $\phi(\dot{\boldsymbol{x}})$ over the dotted variables $\dot{\boldsymbol{x}}$. Without loss of generality, we can restrict $\phi$ to be of the form $A_{1} \dot{\boldsymbol{x}} \leq \boldsymbol{b}_{1} \wedge A_{2} \dot{\boldsymbol{x}} \geq$ $\boldsymbol{b}_{2}$, where $A_{1}, A_{2}$ are $n \times n$ rational matrices and $\boldsymbol{b}_{1}, \boldsymbol{b}_{2}$ are $n \times 1$ vectors consisting of positive rationals. These dynamics can be abstracted by the relation

$$
0 \leq \max \left(A_{1}\left(\boldsymbol{x}-\boldsymbol{x}_{0}\right) \cdot / \boldsymbol{b}_{1}\right) \leq \min \left(A_{2}\left(\boldsymbol{x}-\boldsymbol{x}_{0}\right) \cdot / \boldsymbol{b}_{2}\right)
$$




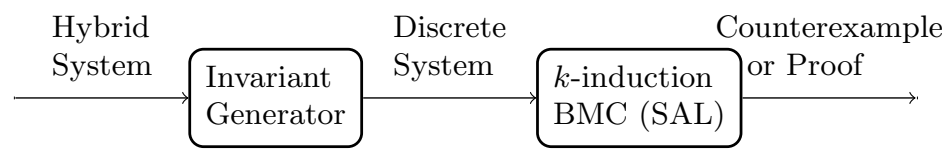

Fig. 2. Framework for implementing a safety verification engine using relationalization

where ./ is componentwise division. This is again a complete abstraction. Note that the result for rectangular automata is obtained as a special case where $A_{1}, A_{2}$ are identity matrices.

Linear Systems with Rational Eigenvalues: First consider the linear system $\frac{d \boldsymbol{x}}{d t}=D \boldsymbol{x}$, where $D$ is a diagonal matrix with rational entries $\lambda_{1}, \ldots, \lambda_{n}$. For simplicity assume $\lambda_{i} \neq 0$ for all $i$. Since they are rational, the $\lambda_{i}$ 's can be written as integer multiples of some base rational $\lambda$, that is, $\lambda_{1}=c_{1} \lambda, \ldots, \lambda_{n}=$ $c_{n} \lambda$ for some rational $\lambda$ and integers $c_{1}, \ldots, c_{n}$. A complete relational abstraction of the linear system is given by

$$
(\exists r>0) \bigwedge_{i=1}^{n}\left[x_{i}=r^{c_{i}} x_{i 0}\right]
$$

If $A$ is not diagonal, but diagonalizable with all rational eigenvalues, then a relational abstraction can be obtained in the same way after doing a change of variables transformation. If $A$ is nilpotent, then again the linear system can be shown to have a complete relational abstraction.

\section{Implementation}

Figure 2 shows the overall verification framework. It consists of two parts: (a) an invariant generator for generating the relational abstraction of the input hybrid system, and (b) a verifier for analyzing the relational abstraction using techniques such as $k$-induction and bounded model checking (BMC). Note that other verification techniques/tools are equally applicable here. Our framework abstracts each mode up front. It is possible, in practice, to implement the abstraction on-the-fly, whenever a previously unseen mode is entered.

We now discuss the implementation of relational abstraction, restricting our attention here to techniques that have been employed in our experiments. We primarily apply template-based methods for generating relational abstractions 919. Template-based techniques formulate an unknown parameterized form for the required invariant and cast the problem of generating the invariant as an $\exists \forall$ formula. These $\exists \forall$ formulas can be solved directly using quantifier elimination techniques over the theory of reals [428], or they can be first converted into $\exists$ formulas through dualization. The $\exists$ formulas, which contain nonlinear constraints over the unknown parameters, can be solved using either fixed point iteration over cones [36], or using bit-vector solvers [19], or by simulating the system numerically 20 . In our experiments, we use a specialized quantifier elimination technique [38] and the tool TimePass, which implements a fixed point 
iteration with widening over polyhedral cones for affine ODEs [36]. We consider three types of abstractions affine, eigen, and box.

Affine abstractions: Affine abstractions employ the template: $\boldsymbol{a} \cdot \boldsymbol{x}+\boldsymbol{b} \cdot \boldsymbol{x}_{0} \geq$ $a_{0}$. In practice, the template: $\boldsymbol{a}\left(\boldsymbol{x}-\boldsymbol{x}_{0}\right) \geq a_{0}$ suffices after taking the initiation into account. Affine relational abstractions are computationally inexpensive to generate, but they are also of relatively poor quality.

Eigen abstractions: For linear systems, such as $d \boldsymbol{x} / d t=A \boldsymbol{x}$, whenever $A$ has real eigenvalues, useful relational abstractions can be generated using the eigenvectors of $A^{T}$ corresponding to those real eigenvalues 39]. Here, $A^{T}$ denotes the transpose of matrix $A$. Specifically, if $\boldsymbol{c}$ is such that $A^{T} \boldsymbol{c}=\lambda \boldsymbol{c}$, then by simple algebraic manipulation, we obtain $\frac{d}{d t}\left(c_{1} x_{1}+\ldots+c_{n} x_{n}\right)=\lambda\left(c_{1} x_{1}+\ldots+c_{n} x_{n}\right)$ where $\boldsymbol{c}:=\left[c_{1} ; \ldots ; c_{n}\right]$ and $\boldsymbol{x}:=\left[x_{1} ; \ldots ; x_{n}\right]$. Let $p$ denote the linear expression $c_{1} x_{1}+\ldots+c_{n} x_{n}$ and let $p_{0}$ denote the linear expression $c_{1} x_{10}+\ldots+c_{n} x_{n 0}$. Here, $x_{i 0}$ denotes the old value of $x_{i}$. If $\lambda<0$, then we know that the value of $p$ approaches zero monotonically. Consequently, we get the relational abstraction $\left(p_{0}<0 \Rightarrow p_{0} \leq p<0\right) \wedge\left(p_{0}>0 \Rightarrow p_{0} \geq p>0\right)$. Similarly, we can write the relational invariants for the case when $\lambda>0$ and $\lambda=0$.

Box abstractions: Box relational abstractions are Boolean combinations of affine relational invariants of the form

$$
\max \left(a_{1}\left|x_{1}\right|, \ldots, a_{n}\left|x_{n}\right|\right) \leq \max \left(a_{1}\left|x_{10}\right|, \ldots, a_{n}\left|x_{n 0}\right|\right)
$$

where $a_{i}$ 's are unknown nonnegative real numbers. Discovering appropriate values for $a_{i}$ s does not require expensive quantifier elimination. We can find box relational invariants in $O\left(n^{3}\right)$ time. Box invariants do not always exist: sufficient (and necessary) conditions for their existence are known [1]. Example 3 shows a box invariant for the switch open mode.

\section{Experimental Evaluation}

We evaluate our approach over the navigation benchmarks [14, to experimentally evaluate the usefulness of relational abstractions for verifying hybrid systems. The navigation benchmarks model a vehicle moving in a 2-dimensional rectangular space $[0, m-1] \times[0, n-1]$. This space is partitioned in $m \times n$ cells. Let $x, y$ denote the position of the vehicle and $v_{x}, v_{y}$ denote its velocity. Then the dynamics of the vehicle in any particular cell are given by the ODEs:

$$
\begin{array}{ll}
\frac{d x}{d t}=v_{x} & \frac{d v_{x}}{d t}=a_{11}\left(v_{x}-b\right)+a_{12}\left(v_{y}-c\right) \\
\frac{d y}{d t}=v_{y} & \frac{d v_{y}}{d t}=a_{21}\left(v_{x}-b\right)+a_{22}\left(v_{y}-c\right)
\end{array}
$$

where the matrix $A:=\left[a_{11}, a_{12} ; a_{21}, a_{22}\right]$ and the direction $(b, c)$ are parameters that can potentially vary (for each of the cells) 2 .

Every benchmark in the suite is specified by fixing the matrix $A$, the number of cells $m \times n$, the direction $(b, c)$ in each cell, and initial intervals for each

\footnotetext{
${ }^{2}$ The matrix $A$ is Hurwitz: the dynamics for $\left(v_{x}, v_{y}\right)$ asymptotically converge to $(b, c)$.
} 
Table 1. Comparison of various abstractions over the NAV benchmarks. All experiments were performed on an Intel Xeon E5630 2.53GHz single-core processor (x86_64 arch) with 4GB RAM running Ubuntu Linux 2.6.32-26. Legend - depth: $k$-induction depth, time: time taken by verifier, status: P: Proved Property, CE: $k$-induction base case fails and counterexample is produced, F: inductive step fails, no proofs or counterexample. Note: Relational eigeninvariants are inapplicable for nav07, nav08 (indicated by -). $k$-induction timings reported as $t_{1}+t_{2}$ indicate that an auxiliary lemma was used. $t_{1}$ is the time to prove the property, and $t_{2}$ to discharge the lemma.

\begin{tabular}{|c||c|c|c||c|c|c||c|c|c|}
\hline \multicolumn{1}{|c||}{ Benchmark } & \multicolumn{3}{c||}{ Affine Invs } & \multicolumn{3}{c||}{ Affine+Eigen Invs } & \multicolumn{2}{c|}{ Affine+Eigen+Box Invs } \\
\hline & depth & status & time(s) & depth & status & time(s) & depth & status & time(s) \\
\hline \hline nav01 & 4 & $\mathrm{~F}$ & 0.63 & 4 & $\mathrm{~F}$ & 0.88 & 4 & $\mathrm{~F}$ & 1.91 \\
nav01 & 5 & $\mathrm{P}$ & 0.75 & 5 & $\mathrm{P}$ & 0.91 & 5 & $\mathrm{P}$ & 1.36 \\
\hline nav02 & 4 & $\mathrm{~F}$ & 0.64 & 4 & $\mathrm{~F}$ & 0.87 & 4 & $\mathrm{~F}$ & 1.8 \\
nav02 & 5 & $\mathrm{P}$ & 0.68 & 5 & $\mathrm{P}$ & 1.04 & 5 & $\mathrm{P}$ & 3.33 \\
\hline nav03 & 4 & $\mathrm{~F}$ & 0.60 & 4 & $\mathrm{~F}$ & 0.91 & 4 & $\mathrm{~F}$ & 1.72 \\
nav03 & 5 & $\mathrm{P}$ & 0.67 & 5 & $\mathrm{P}$ & 1.05 & 5 & $\mathrm{P}$ & 2.7 \\
\hline nav04 & 3 & $\mathrm{CE}$ & 0.49 & 8 & $\mathrm{~F}$ & 3.21 & 8 & $\mathrm{~F}$ & 34.883 \\
nav04 & & & & 4 & $\mathrm{P}$ & $0.75+0.99$ & 4 & $\mathrm{P}$ & $0.98+2.21$ \\
\hline nav05 & 2 & $\mathrm{CE}$ & 0.47 & 8 & $\mathrm{~F}$ & 3.85 & 8 & $\mathrm{~F}$ & 37.31 \\
nav05 & & & & 8 & $\mathrm{P}$ & $2.15+2.50$ & 8 & $\mathrm{P}$ & $5.38+11.05$ \\
\hline nav06 & 4 & $\mathrm{CE}$ & 0.61 & 8 & $\mathrm{~F}$ & 18.01 & 8 & $\mathrm{~F}$ & 494.5 \\
nav06 & 4 & $\mathrm{CE}$ & 1.03 & 8 & $\mathrm{P}$ & $21.80+7.42$ & 8 & $\mathrm{P}$ & $40.22+35.08$ \\
\hline nav07 & 5 & $\mathrm{CE}$ & 0.66 & - & - & - & 5 & $\mathrm{~F}$ & 69.9 \\
nav07 & & & & - & - & - & 6 & $\mathrm{P}$ & 6.25 \\
\hline nav08 & 4 & $\mathrm{CE}$ & 0.52 & - & - & - & 6 & $\mathrm{CE}$ & 0.95 \\
\hline nav09 & 4 & $\mathrm{CE}$ & 0.57 & 4 & $\mathrm{CE}$ & 1.45 & 4 & $\mathrm{CE}$ & 19.87 \\
\hline nav10 & 3 & $\mathrm{CE}$ & 0.44 & 3 & $\mathrm{CE}$ & 0.99 & 3 & $\mathrm{CE}$ & 0.95 \\
\hline
\end{tabular}

of the four state variables $x, y, v_{x}, v_{y}$. Our experiments focus on proving the unreachability of a distinct cell marked $B$ for each benchmark instance 14.

In our experiments, we verify the safety property for the navigation benchmarks using $k$-induction over the relational abstraction. We use the SAL infinite bounded model checker, with the $k$-induction flag turned on (sal-inf-bmc -i), which uses the SMT solver Yices in the back end. Table1reports the results. For each benchmark, we report the depth used for performing $k$-induction (under "depth"), the output of $k$-induction (under "status"), and the time it took (under "time"). There are three possible outputs: (a) the base case of $k$-induction fails and a counterexample is found (denoted by "CE"), (b) the base case is proved, but the induction step fails; i.e., no counterexample is found, but no proof is found either (denoted by " $F$ "), (c) the base case and the induction step are successfully proved (denoted by "P"). Since we perform $k$-induction on an abstraction, the counterexamples may be spurious, but the proofs are not. As Table 1 indicates, relational abstractions are sufficient to establish safety of the benchmarks nav01-nav05, nav06*, and nav07. The system nav06* is the same as nav06 but with a slightly smaller set of initial states. However, the proof fails on nav06 and nav08-nav10. There are two reasons for failure: (a) poor quality of abstraction, which is reflected in entries "CE" in Table 1, and (b) inability 
to find suitable k-inductive lemmas. This happens in the case of nav06, where the proof fails without yielding a counterexample. As discussed in Section 4, we employed three kinds of relational abstractions for each mode: affine, eigen, and box. Table 1 also shows performance of each of these techniques.

Affine abstractions: In Table 1. Columns (2)-(4) report results on affine relational abstractions. We note that affine abstractions are sufficient to prove safety of benchmarks nav01-nav03, but they fail on all other benchmarks.

Eigen abstractions: The dynamics in each mode of the benchmarks nav01nav06 and nav10 have negative real eigenvalues. In Table 1, Columns (5)-(7) present results using a relational abstraction obtained by combining affine and eigen abstractions. For nav04-nav06, the combination eliminates the spurious counterexamples. However, no such benefit is seen on nav08-nav10 benchmarks. The dynamics in benchmarks nav07-nav08 do not have any real eigenvalues.

Box abstractions: The dynamics of all modes of all benchmarks in Table 1 satisfy all the conditions for the existence of box invariants, which enables us to generate box relational invariants for each of them. Columns (8)-(10) report results using a relational abstraction obtained by combining affine, eigen, and box relational invariants. In the case of nav07, where there are no eigen invariants, addition of box invariants eliminated the counterexamples from the model and even enabled verification of safety using $k$-induction with depth 6 . However, no such benefit is seen for benchmarks nav08-nav10. Also, when eigen invariants exist, then adding box invariants does not seem to improve the quality of abstraction. Note that the use of box invariants increases the time taken to perform $k$-induction: this is expected since box invariants have a complex Boolean structure, which increases the search space of the SMT solver.

Comparison with Other Tools: Comparing our timings with those reported in the literature for the very same benchmarks, especially previous work by one of the authors [35, we note that our techniques are at least an order of magnitude faster on the larger benchmarks (10s of seconds vs. $100 s-1000 s$ of seconds using template-based flowpipes [35]). A detailed comparison will be made available in our extended version.

Disjunctive and conjunctive relational invariants: One plausible reason for the failure to prove nav08-nav10 benchmarks is that we do not consider invariants of richer Boolean structure, such as 2-disjunctive invariants of the form $p\left(\boldsymbol{x}_{0}\right) \geq 0 \Rightarrow p\left(\boldsymbol{x}, \boldsymbol{x}_{0}\right) \geq 0$. Even though eigen invariants have this form, there may be other invariants of this form that are not related to the eigenvectors of the $A$-matrix. We also do not consider conjunctive invariants of the form $p_{1} \geq 0 \wedge p_{2} \geq 0$. Note that $p_{1} \geq 0$ and $p_{2} \geq 0$ need not separately be inductive, but their conjunction could be inductive. For this reason, we often fail to find them by just considering templates for $p_{1} \geq 0$ and $p_{2} \geq 0$ separately.

Overcoming limitations of $k$-induction: Even if the relational abstractions are strong enough to rule out all unsafe behaviors, we may still fail to prove the system safe using $k$-induction. This will happen if the safety property is not $k$-inductive for any $k$. This is possibly the case for benchmarks nav04-nav06. 
Table 2. Time (in milliseconds) to generate all affine equality, inequality and eigen relational invariants for all modes of all the benchmarks

\begin{tabular}{|c||c|c|}
\hline Type of Relational Invariant & Time (no state invariant) & Time (with state invariant) \\
\hline \hline Affine Inequality & $60 \mathrm{~ms}$ & $6740 \mathrm{~ms}$ \\
\hline Affine Equality + Eigen & $70 \mathrm{~ms}$ & $340 \mathrm{~ms}$ \\
\hline
\end{tabular}

However, we are able to successfully prove safety of nav04 and nav05 by using an auxiliary lemma. The auxiliary lemma was itself verified by $k$-induction again. For nav06, we are unable to find any suitable auxiliary lemma at this time.

Another plausible cause for the failure of $k$-induction is the introduction of spurious loops in the relational abstraction, where no such loops exist in the concrete system. Analysis of the counterexamples to the induction step in nav06 (generated by sal-inf-bmc -i -ice) strongly indicates this possibility.

One way to eliminate spurious loops in the abstract is based on assuming that the concrete system stays in a mode for some small, but fixed, amount of time. Under the assumption that the concrete system stayed in a mode for at least 0.1 second, we strengthened the affine invariants of nav06, allowing us to prove safety of nav06 (for a slightly smaller set of initial states than what is specified in the nav06 benchmarks). These results are reported in row nav06* in Table 1 . We conjecture that this trick will eliminate all the spurious counterexamples in the other navigation benchmarks.

Quantifier elimination for generating relational invariants: The new redlog/qepcad combination [38] is quite effective in generating all the affine and eigen invariants used in our experiments. Table 2 provides the time taken by all runs of the quantifier elimination process to generate these invariants. We report times for two cases depending on whether we used a template of the form $\psi\left[\boldsymbol{x}_{0}\right] \Rightarrow$ $R\left(\boldsymbol{x}_{0}, \boldsymbol{x}\right)$, with a state invariant antecedent guarding the relation. The times are negligible since the benchmarks are 4-dimensional systems (they all involve only four real-valued variables) with relatively simple (linear) dynamics in each mode. As a final remark, note that quantifier elimination does not return specific values for the parameters, but constraints on the unknown parameters. We choose values by solving a satisfiability problem. In our examples, the constraints after elimination were simple enough to perform this step manually. The redlog files that were used to generate the relational invariants and SAL models of the relational abstraction are publicly available 3 .

\section{Conclusions}

We have presented an approach for verifying hybrid systems based on relational abstractions. Relational abstractions can be constructed compositionally by abstracting each mode separately. Our initial results are quite encouraging. The technique successfully solves some of the standard benchmark examples an order of magnitude faster than symbolic model checkers. The abstractions can be

3 http://www.csl.sri.com/ tiwari/relational-abstraction/ 
coarse, and $k$-induction itself can be challenging to apply on hybrid systems in practice. Our future work will focus on improving the speed and precision of relational abstraction generation to enable fast proofs for complex systems. We also wish to apply our techniques to nonlinear hybrid systems in order to derive linear arithmetic abstractions.

\section{References}

1. Abate, A., Tiwari, A., Sastry, S.: Box invariance in biologically-inspired dynamical systems. Automatica 45(7), 1601-1610 (2009)

2. Alur, R., Dang, T., Ivančić, F.: Counter-example guided predicate abstraction of hybrid systems. In: Garavel, H., Hatcliff, J. (eds.) TACAS 2003. LNCS, vol. 2619, pp. 208-223. Springer, Heidelberg (2003)

3. Asarin, E., Dang, T., Girard, A.: Hybridization methods for the analysis of nonlinear systems. Acta Informatica 43, 451-476 (2007)

4. Berdine, J., Chawdhary, A., Cook, B., Distefano, D., O'Hearn, P.W.: Variance analyses from invariance analyses. In: POPL, pp. 211-224. ACM, New York (2007)

5. Berz, M., Makino, K.: Performance of Taylor Model Methods for Validated Integration of ODEs. In: Dongarra, J., Madsen, K., Waśniewski, J. (eds.) PARA 2004. LNCS, vol. 3732, pp. 65-73. Springer, Heidelberg (2006)

6. Blanchini, F., Miani, S.: Set-Theoretic Methods in Control. Springer, Heidelberg (2008)

7. Clarke, E.M., Grumberg, O., Jha, S., Lu, Y., Veith, H.: Counterexample-guided abstraction refinement for symbolic model checking. J. ACM 50(5), 752-794 (2003)

8. Collins, G.E., Hong, H.: Partial cylindrical algebraic decomposition for quantifier elimination. Journal of Symbolic Computation 12(3), 299-328 (1991)

9. Colón, M.A., Sankaranarayanan, S., Sipma, H.B.: Linear invariant generation using non-linear constraint solving. In: Hunt Jr., W.A., Somenzi, F. (eds.) CAV 2003. LNCS, vol. 2725, pp. 420-432. Springer, Heidelberg (2003)

10. Colón, M.A., Sipma, H.B.: Synthesis of linear ranking functions. In: Margaria, T., Yi, W. (eds.) TACAS 2001. LNCS, vol. 2031, pp. 67-81. Springer, Heidelberg (2001)

11. Cousot, P., Cousot, R.: Abstract Interpretation: A unified lattice model for static analysis of programs by construction or approximation of fixpoints. In: ACM Principles of Programming Languages, pp. 238-252 (1977)

12. Dang, T., Maler, O., Testylier, R.: Accurate hybridization of nonlinear systems. In: HSCC 2010, pp. 11-20. ACM, New York (2010)

13. Dang, T., Salinas, D.: Image Computation for Polynomial Dynamical Systems Using the Bernstein Expansion. In: Bouajjani, A., Maler, O. (eds.) CAV 2009. LNCS, vol. 5643, pp. 219-232. Springer, Heidelberg (2009)

14. Fehnker, A., Ivančić, F.: Benchmarks for hybrid systems verification. In: Alur, R., Pappas, G.J. (eds.) HSCC 2004. LNCS, vol. 2993, pp. 326-341. Springer, Heidelberg (2004)

15. Frehse, G.: PHAVer: Algorithmic Verification of Hybrid Systems Past HyTech. STTT 10(3) (June 2008)

16. Girard, A.: Reachability of uncertain linear systems using zonotopes. In: Morari, M., Thiele, L. (eds.) HSCC 2005. LNCS, vol. 3414, pp. 291-305. Springer, Heidelberg (2005) 
17. Guernic, C.L., Girard, A.: Reachability analysis of linear systems using support functions. Nonlinear Analysis: Hybrid Systems 4(2), 250-262 (2010)

18. Gulwani, S., Jain, S., Koskinen, E.: Control-flow refinement and progress invariants for bound analysis. In: PLDI (2009)

19. Gulwani, S., Tiwari, A.: Constraint-based approach for analysis of hybrid systems. In: Gupta, A., Malik, S. (eds.) CAV 2008. LNCS, vol. 5123, pp. 190-203. Springer, Heidelberg (2008)

20. Gupta, A., Majumdar, R., Rybalchenko, A.: From tests to proofs. In: Kowalewski, S., Philippou, A. (eds.) TACAS 2009. LNCS, vol. 5505, pp. 262-276. Springer, Heidelberg (2009)

21. Halbwachs, N., Proy, Y.-E., Roumanoff, P.: Verification of real-time systems using linear relation analysis. In: FMSD, vol. 11(2), pp. 157-185 (1997)

22. Henzinger, T.A.: The theory of hybrid automata. In: LICS 1996, pp. 278-292. IEEE, Los Alamitos (1996)

23. Henzinger, T.A., Ho, P.-H., Wong-Toi, H.: Algorithmic analysis of nonlinear hybrid systems. IEEE Transactions on Automatic Control 43, 540-554 (1998)

24. Kurzhanski, A.B., Varaiya, P.: Ellipsoidal techniques for reachability analysis. In: Lynch, N.A., Krogh, B.H. (eds.) HSCC 2000. LNCS, vol. 1790, pp. 202-214. Springer, Heidelberg (2000)

25. Meiss, J.D.: Differential Dynamical Systems. SIAM publishers, Philadelphia (2007)

26. Mysore, V., Piazza, C., Mishra, B.: Algorithmic algebraic model checking II: Decidability of semi-algebraic model checking and its applications to systems biology. In: Peled, D.A., Tsay, Y.-K. (eds.) ATVA 2005. LNCS, vol. 3707, pp. 217-233. Springer, Heidelberg (2005)

27. Nielson, F., Nielson, H.R., Hankin, C.: Principles of Program Analysis. Springer, Heidelberg (1999)

28. Oishi, M., Mitchell, I., Bayen, A.M., Tomlin, C.J.: Invariance-preserving abstractions of hybrid systems: Application to user interface design. IEEE Trans. on Control Systems Technology 16(2) (March 2008)

29. Platzer, A., Clarke, E.: Computing differential invariants of hybrid systems as fixedpoints. Formal Methods in Systems Design 35(1), 98-120 (2009)

30. Podelski, A., Rybalchenko, A.: Transition invariants. In: LICS, pp. 32-41. IEEE, Los Alamitos (2004)

31. Podelski, A., Wagner, S.: Model checking of hybrid systems: From reachability towards stability. In: Hespanha, J.P., Tiwari, A. (eds.) HSCC 2006. LNCS, vol. 3927, pp. 507-521. Springer, Heidelberg (2006)

32. Prajna, S., Jadbabaie, A.: Safety verification of hybrid systems using barrier certificates. In: Alur, R., Pappas, G.J. (eds.) HSCC 2004. LNCS, vol. 2993, pp. 477-492. Springer, Heidelberg (2004)

33. Ratschan, S., She, Z.: Safety verification of hybrid systems by constraint propagation based abstraction refinement. In: Morari, M., Thiele, L. (eds.) HSCC 2005. LNCS, vol. 3414, pp. 573-589. Springer, Heidelberg (2005)

34. Rushby, J., Lincoln, P., Owre, S., Shankar, N., Tiwari, A.: Symbolic analysis laboratory (SAL). Cf, http://www.csl.sri.com/projects/sal/

35. Sankaranarayanan, S., Dang, T., Ivančić, F.: Symbolic model checking of hybrid systems using template polyhedra. In: Ramakrishnan, C.R., Rehof, J. (eds.) TACAS 2008. LNCS, vol. 4963, pp. 188-202. Springer, Heidelberg (2008)

36. Sankaranarayanan, S., Sipma, H.B., Manna, Z.: Fixed point iteration for computing the time elapse operator. In: Hespanha, J.P., Tiwari, A. (eds.) HSCC 2006. LNCS, vol. 3927, pp. 537-551. Springer, Heidelberg (2006) 
37. Sheeran, M., Singh, S., Stålmarck, G.: Checking safety properties using induction and a SAT-solver. In: Johnson, S.D., Hunt Jr., W.A. (eds.) FMCAD 2000. LNCS, vol. 1954, pp. 108-125. Springer, Heidelberg (2000)

38. Sturm, T., Tiwari, A.: Verification and synthesis using real quantifer elimination (2011) (submitted)

39. Tiwari, A.: Approximate reachability for linear systems. In: Maler, O., Pnueli, A. (eds.) HSCC 2003. LNCS, vol. 2623, pp. 514-525. Springer, Heidelberg (2003)

40. Tiwari, A.: HybridSAL: A tool for abstracting HybridSAL specifications to SAL specifications (2007)

41. Tiwari, A.: Abstractions for hybrid systems. Formal Methods in Systems Design 32, 57-83 (2008)

42. Weispfenning, V. In: Applied Algebra and Error-Correcting Codes (AAECC) 